\title{
New Strategies to Improve Patient Adherence to Medications for Noncommunicable Diseases During and After the COVID-I 9 Era Identified via a Literature Review
}

\author{
Tarek A Hassan' \\ Jorge Enrique Sáenz ${ }^{2}$ \\ Danute Ducinskiene ${ }^{3}$ \\ Joseph P Cook' \\ Joseph S Imperato (ID) \\ Kelly H Zou (D) \\ 'Global Medical and Clinical, Viatris, \\ Canonsburg, PA, USA; ${ }^{2}$ Medical, Viatris, \\ Madrid, Spain; ${ }^{3}$ Medical Affairs, Viatris, \\ Vilnius, Lithuania
}

\begin{abstract}
Noncommunicable diseases (NCDs) place a huge burden on healthcare systems and society as a whole. Relatively early in the coronavirus disease 2019 (COVID-19) pandemic, clinicians became aware that in individuals infected with COVID-19, those with preexisting NCDs such as diabetes mellitus and cardiovascular disease (CVD) were at a greater risk of poor outcomes and mortality than those without. The importance of adherence to medications and lifestyle changes to control and prevent NCDs has been a major focus for many years, but with limited success - the proportion of patients adherent and persistent to their medications remains very low. There are many facets to adherence and persistence. Recent evidence suggests that a patient-centric approach is important, and ensuring that a patient is both motivated and empowered is critical to improving adherence/persistence. The COVID-19 pandemic has brought many changes to the way in which patients with NCDs are managed, with telemedicine and ehealth becoming more common. Changes have also occurred in the way in which patients can gain access to medications during the pandemic. The potential for these changes forms the basis of improving the management of patients with NCDs both during and after the pandemic. Over the coming months, a huge amount of work will be put into initiatives to promote adherence to COVID19 vaccination programs. Those at highest risk of severe COVID-19, such as people aged 80 years and older, are likely to receive the vaccine first in some parts of world. Finally, social determinants of health are critical elements that can impact not just the likelihood of having an NCD or becoming infected with COVID-19, but also access to healthcare, and a patient's adherence and persistence with their treatments.
\end{abstract}

Keywords: patients, adherence, persistence, noncommunicable diseases, COVID-19, telemedicine, ehealth

\section{Introduction}

Noncommunicable diseases (NCDs) are defined as diseases that are not passed from person to person (ie, non-infectious), are of long duration (ie, chronic), and result from a combination of genetic, physiological, environmental, and behavioral factors such as tobacco use, an unhealthy diet, physical inactivity, and the harmful use of alcohol. ${ }^{1}$ Metabolic syndrome risk factors (ie, raised blood pressure [BP], being overweight/ obesity, hyperglycemia, hyperlipidemia) also increase the risk of acquiring NCDs. ${ }^{1}$ The fact that many NCDs have the same or similar risk factors means that they often
Correspondence: Kelly H Zou

Global Medical and Clinical, Viatris, 1000 Mylan Blvd, Canonsburg, PA, I5317, USA

Email Kelly.Zou@viatris.com 
co-occur in the same individual, ${ }^{2}$ which both adds to the burden of, and complicates the management of NCDs.

All healthcare professionals (HCPs) are acutely aware of the need to prevent and manage NCDs such as cardiovascular disease (CVD) and cancer. However, many HCPs are not yet familiar with the term NCD, or what diseases are categorized as NCDs, despite them being a leading cause of mortality and morbidity. For example, in the USA, NCDs are estimated to account for $88 \%$ of all deaths, with four categories of NCDs being responsible for the majority of these deaths: CVD, cancer, chronic respiratory disease, and diabetes (Figure 1). ${ }^{3}$ The financial burden associated with NCDs is also vast. For example, it has been estimated that in the USA the costs of managing NCDs and reduced productivity due to NCDs will reach $\$ 4.2$ trillion by 2023 . $^{4}$

Screening, detection, prevention, and treatment of NCDs, as well as palliative care, are key components of the successful management of NCDs. ${ }^{1}$ The World Health Organization (WHO) has called for programs aimed at preventing NCDs to be implemented through targeting modifiable risk factors, such as tobacco use, the harmful use of alcohol, physical inactivity, and unhealthy diets. ${ }^{5}$ With this in mind, the WHO has set a series of preventative targets to be achieved by 2025 (Figure 2). ${ }^{5}$ These preventative programs are more cost-effective than treating the consequence of NCDs. For example, it has been estimated that every US $\$ 1$ spent on preventing NCDs will have a return of at least US\$7 in the following 10 years. ${ }^{5}$ This paper focuses on key areas involved in NCDs - that is medications and interventions aimed at reducing the impact/burden of NCDs - notably ensuring that patients are adherent with their prescribed treatment regimens. In addition, the impact of COronaVIrus Disease 2019 (COVID-19) on both patients with NCDs and on their adherence with treatments is discussed.

A series of literature searches were conducted in the preparation of this paper. Search terms included: "access", "patients adherence", "compliance", "persistence", "reimbursement", "COVID-19", "coronavirus", "telemedicine", "noncommunicable diseases", "cardiovascular disease", "patient-centric", and/or "sustainability". A combination of peer-reviewed publications and gray literature were included in this process. For example, using the PubMed database, a comprehensive search for "patients' adherence" resulted in 150,494 abstracts and manuscripts that were subject for inclusion and exclusion in further review. The Medication Adherence and Persistence special interest group's work by

\section{Proportional Mortality}

NCDs are estimated to account for $\mathbf{8 8 \%}$ of all deaths

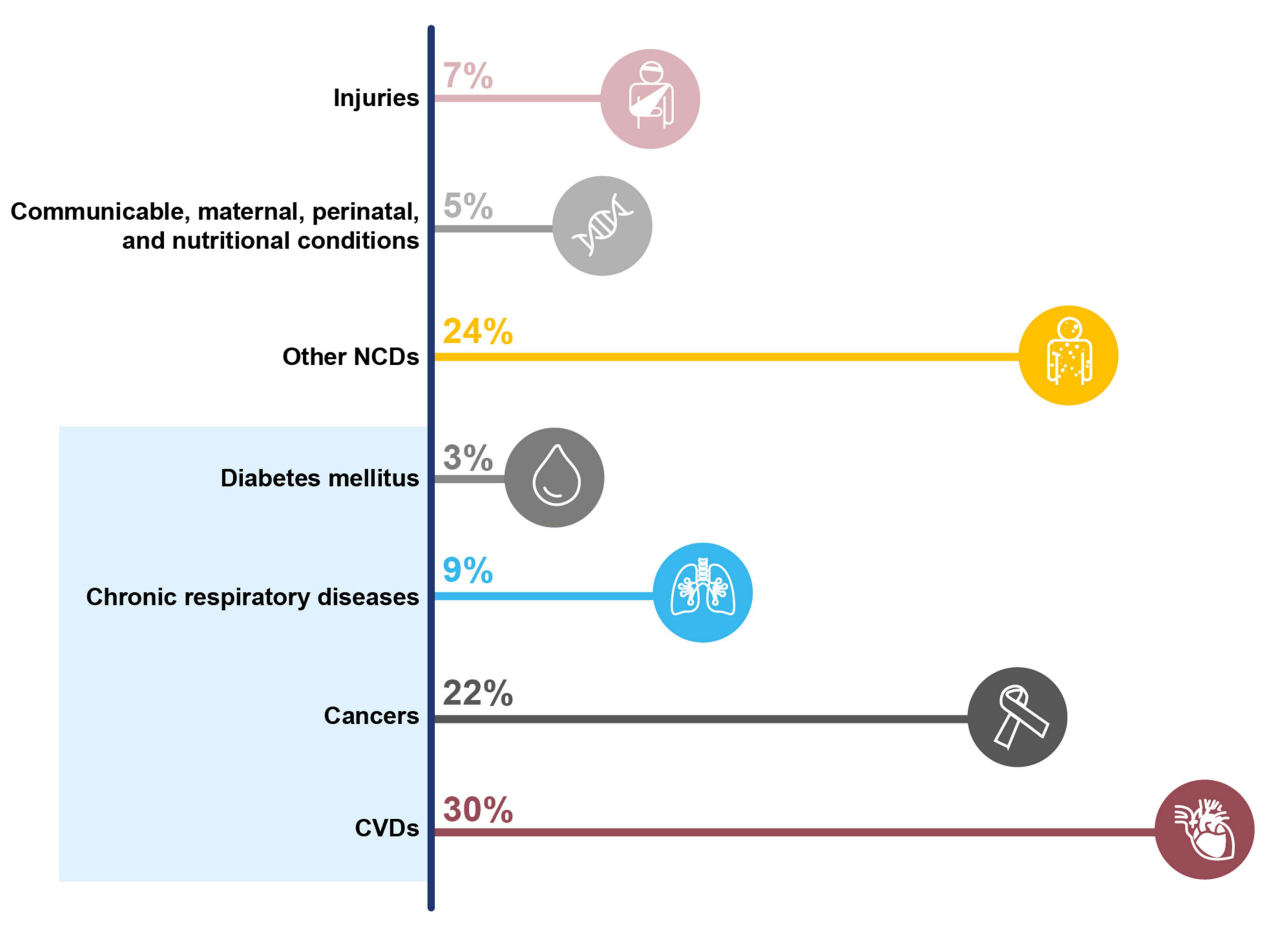

Figure I Mortality by disease groups in the USA during 2016. Data from World Health Organization. ${ }^{3}$ Abbreviations: CVD, cardiovascular disease; NCD, noncommunicable disease. 


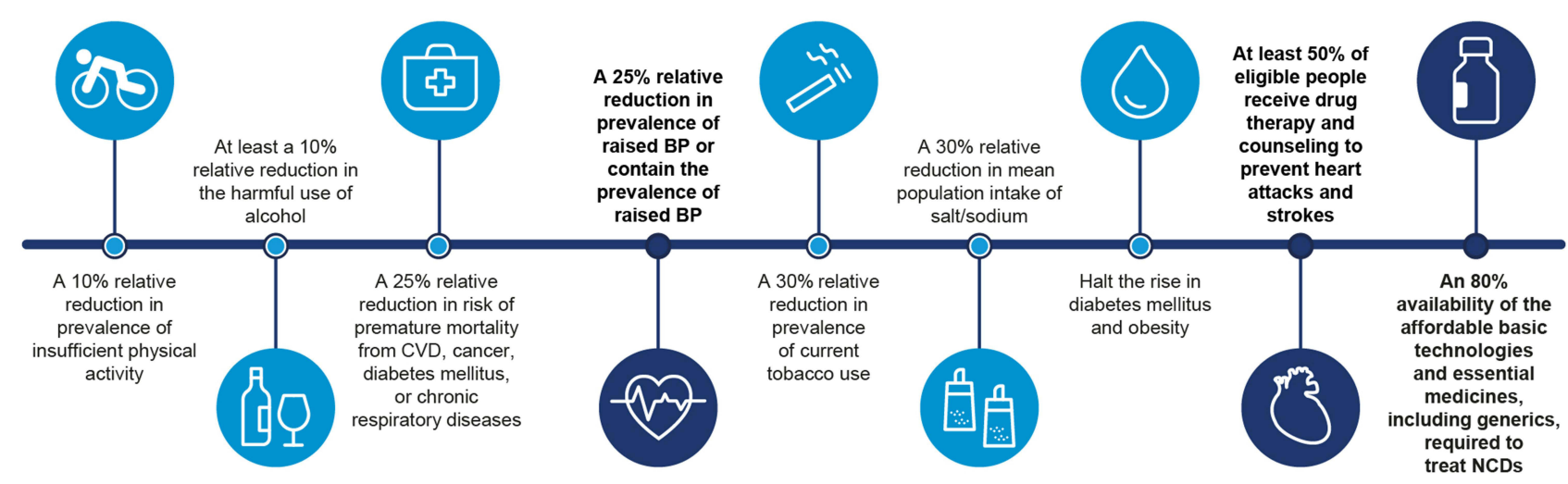

Figure 2 World Health Organization's nine voluntary global targets for NCDs to be achieved by 2025, with baseline values from 20I0. Data from World Health Organization. ${ }^{97}$

Abbreviations: BP, blood pressure; CVD, cardiovascular disease; NCD, noncommunicable disease.

the International Society for Pharmacoeconomics and Outcomes Research was also carefully reviewed. ${ }^{6}$

Before focusing on adherence, it is important to provide details on the relationship between NCDs and COVID-19 and how this might have impacted patient adherence with medications used to manage NCDs. Since first being identified and named at the start of 2020, as of June 9, 2021, there have been over 174 million confirmed COVID-19 cases globally, and more than 3.7 million people have died in relation to this condition. ${ }^{7}$ Numerous studies have demonstrated that NCDs are frequently comorbid conditions in those with severe COVID-19. ${ }^{8-13}$
Studies conducted across a range of regions and settings have demonstrated that NCDs both worsen the clinical outcomes and increase the risk of death among those infected with COVID-19 (Figure 3). ${ }^{9,12,14-18}$ For example, in Italy among hospitalized patients dying with COVID$19,36 \%$ had type 2 diabetes and $30 \%$ had ischemic heart disease; and in Spain 51\% of those with severe COVID-19 had preexisting CVD. ${ }^{19}$ Furthermore, in a survey conducted by the WHO in May 2020, 75\% of countries reported that NCD care services had been disrupted during the pandemic. ${ }^{19}$ Therefore, managing and preventing NCDs is highly important. Evidence is also accumulating

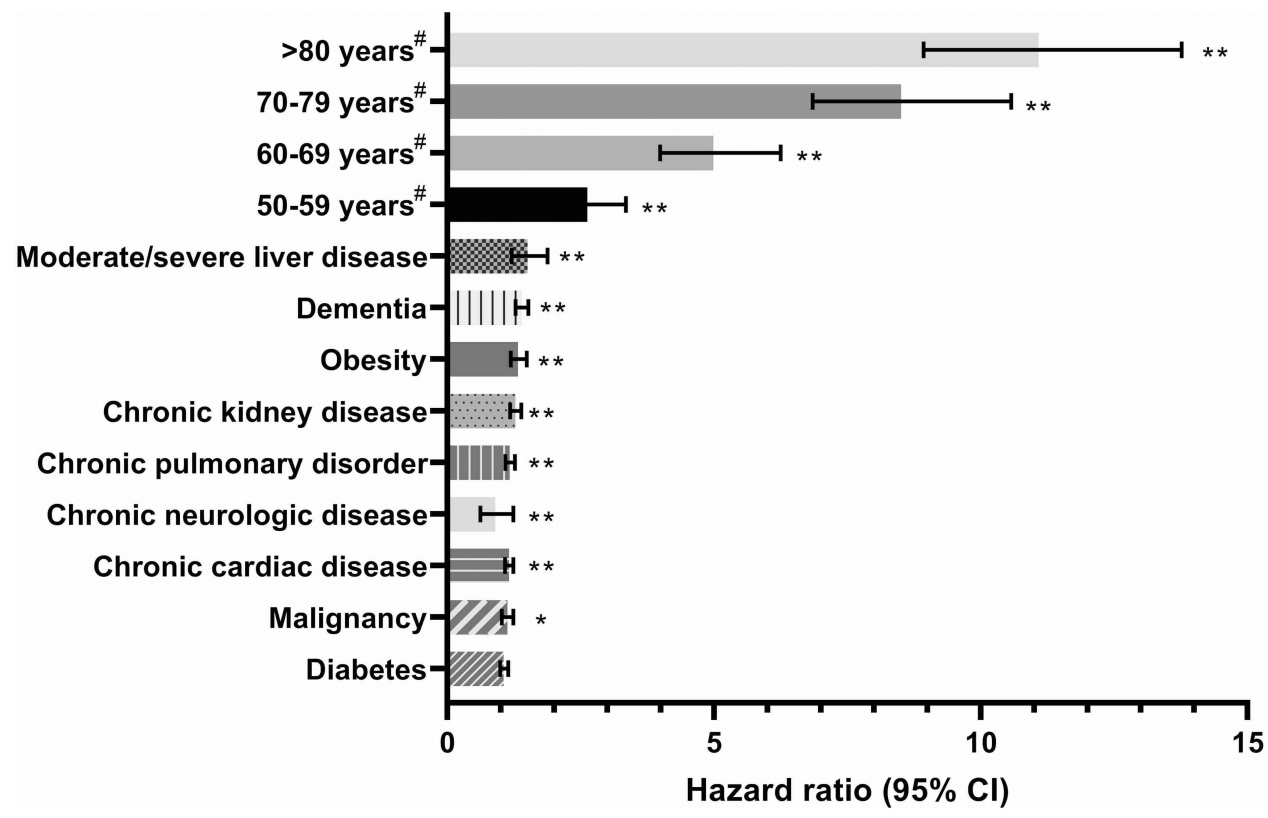

Figure 3 Comorbid NCDs increase the risk of death in patients with COVID-19. ${ }^{9}$ Multivariate analysis of patient characteristics associated with mortality in hospital. Based on 20,133 UK patients with COVID-19. " Patient age was compared against $<50$ years. $* P<0.05, * * P<0.01$. Data from Docherty et al. ${ }^{9}$

Abbreviations: $\mathrm{Cl}$, confidence interval; COVID-19, coronavirus disease 2019. 
to suggest that improving the management, treatment, and/ or control of NCDs, including diabetes, hypertension, or dyslipidemia, may lead to better clinical outcomes in those infected with COVID-19. ${ }^{20-23}$ Indeed, there is some evidence that during the COVID-19 pandemic, more individuals successfully stopped smoking than before the pandemic. $^{24}$

Social determinants of health are increasingly being recognized as playing an important role in the development of NCDs. ${ }^{25,26}$ Those living in more socially deprived areas are more likely to have NCDs, or risk factors for NCDs such as obesity and exposure to air pollution, than those living in more affluent areas. ${ }^{25-27}$ In the past year since early 2020, this relationship between social deprivation and high prevalence of NCDs has been brought into sharp focus in countries that have had high levels of COVID-19 infections. ${ }^{28}$ Those who live in areas of economic deprivation are at a greater risk than those who live in more affluent area of both becoming infected with COVID-19 and going on to develop more severe disease. $^{29,30}$

An understanding of the terminology and methods used to assess adherence and interventions designed to improve adherence and persistence is an important prerequisite for those attempting to measure or improve this variable. The term adherence has been defined by the International Society for Pharmacoeconomics and Outcomes Research Medication Compliance and Persistence (ISPOR) Special Interest Group ${ }^{31}$ as the "degree or extent of conformity to the recommendations about day-to-day treatment by the provider with respect to the timing, dosage, and frequency" and the "extent to which a patient acts in accordance with the prescribed interval, and dose of a dosing regimen." 31 In essence, adherence is the number of doses taken as prescribed. The term "compliance" has a similar definition to adherence but is now seldom used due to the implication that the patient is showing obedience to the physician's authority rather than a collaborative partnership being formed between the patient and physician. ${ }^{32}$

Adherence is often measured using the medication possession ratio (MPR), which is calculated using: [sum of days' supply] divided by [the number of days between the first and last fills] plus [the days' supply of last fill]. ${ }^{31,33}$ Alternatively, adherence can be measured using the proportion of days covered (PDC), which is calculated using: [number of days with drug on hand] divided by [the number of days in a specified time period]. ${ }^{31,33}$ Medication adherence is measured over a period of time and reported as a percentage.

The term persistence is defined as the "act of continuing the treatment for the prescribed duration." In addition it is defined as the "duration of time from initiation to discontinuation of therapy." 31 In short, persistence is the number of days a patient takes medication without exceeding a set grace period. Persistence is reported as a continuous variable in terms of number of days for which the therapy was available. Therefore, it is important to assess both adherence and persistence. This is because clinical outcomes are affected not only by how well patients take their medications but also by how long they take them.

The term concordance, which describes the relationship or interaction between the patient and the prescriber, has recently become more commonly used. This term recognizes that the views and beliefs of the patient are vitally important in defining the subsequent therapeutic alliance with the prescriber. $^{34}$ Therefore, concordance describes the modern, patient-centered view of adherence.

Unfortunately, adherence, persistence or concordance to medications used to treat chronic NCDs is frequently poor. ${ }^{35-41}$ Indeed, the WHO has estimated that adherence with long-term therapy for chronic illnesses averages 50\% in developed countries and is even lower in developing countries. $^{35}$ Furthermore, it has been estimated that $20 \%$ of new prescriptions are never filled, and even among those filled, approximately $50 \%$ are taken incorrectly (errors regarding dosage and timing or frequency administration). ${ }^{42}$ Effective treatments are therefore failing to realize their potential in reducing the burden of NCDs owing to poor patient adherence. ${ }^{35}$

The impact of poor adherence and persistence increases as the prevalence of NCDs rises and this factor is likely to be exacerbated further in the COVID-19 pandemic due to the disruption of many elements of the healthcare system, which are reviewed in detail below. Poor patient treatment adherence increases the mortality and morbidity burden and the healthcare utilization and cost of NCDs. ${ }^{37,38,43-45}$ The direct medical costs associated with poor adherence with medications is high, with estimates ranging from $\$ 100$ to $\$ 300$ billion each year in the USA alone. ${ }^{46,47}$ Although there is no direct information on exactly how NCDs contribute to this burden, much of these costs will be due to treatments for NCDs, due to their high prevalence in the USA and chronic course. Improved adherence and persistence with 
medications to manage NCDs is not only associated with improved beneficial clinical outcomes, ${ }^{48}$ but it has also been associated with improved patient quality of life $(\mathrm{QoL})^{49}$ and reduced healthcare costs. ${ }^{50}$

\section{The Importance of a Patient-Centric Approach to Improving Adherence and Persistence}

A detailed understanding of the factors that can influence and impact adherence is needed to develop appropriate strategies to improve adherence with medications. The WHO believes that there are five dimensions of adherence (Figure 4): $:^{35}$

1. Patient-related factors, such as age, sex, ethnicity, comorbidities with medical history, health beliefs, education or knowledge, psychological profile, and belief or lack of belief in treatment. ${ }^{42,51}$ Understanding where a patient is going to obtain information on NCDs or COVID-19 is also important and falls under this dimension. For example, younger people are more likely to obtain

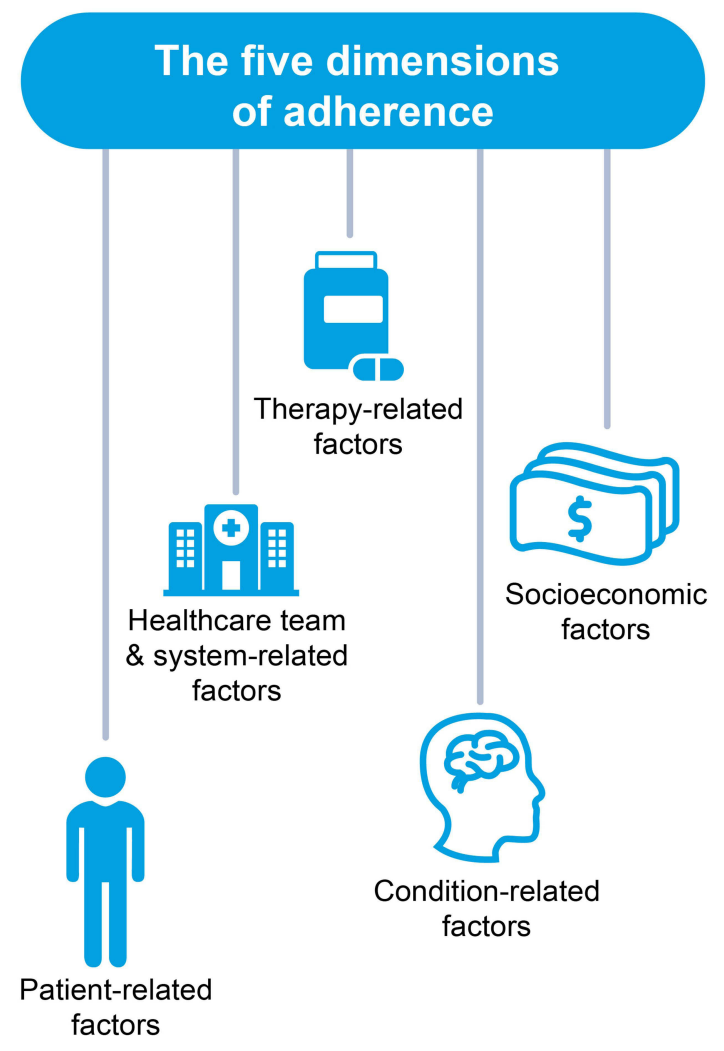

Figure 4 The five dimensions of adherence. Data from World Health Organization. ${ }^{35}$

Abbreviation: HCT, healthcare team. information from social media even when national, government-produced information is available. ${ }^{52}$ Forgetting to take medications or new prescription fills and refills (unintentional factors) or actively deciding to stop treatment or modifying dosing are additional patient-related factors. ${ }^{53}$

2. Healthcare system-related factors, which include reliability of medication supply, availability of healthcare providers or access to healthcare facility, number of outpatient visits, clarity of information regarding medication dosing or administration, and communication between patients and healthcare providers. ${ }^{51}$

3. Therapy-related factors, eg, mode of administration or complexity of dosing regimen, efficacy or effectiveness, tolerability or adverse effects, and complexity of treatment regimen. ${ }^{51}$ These factors should be considered early in the drug development process.

4. Disease or condition-related factors including absence of or improvement of symptoms, disease severity, cognitive impairment, depression, duration of disease, and prognosis. ${ }^{42,51}$

5. Socioeconomic factors, such as cost of medication and copayment, financial and employment status, level of health insurance, stigma of the disease, and family or social support. ${ }^{42,51}$ These are also important factors in both the development of NCDs and the risk of being infected with COVID-19.

Using interventions designed to improve adherence or persistence, and/or the effectiveness of these programs can have a far greater impact on the health of a population than any improvement in specific medical treatments. ${ }^{35}$ Interventions that improve adherence with medications can provide a significant positive return on investment. ${ }^{54}$ This can take the form of both the primary prevention of NCDs (focusing on risk factors) ${ }^{55}$ and secondary prevention of adverse health outcomes, such as lowering the risk of stroke or other CV events. ${ }^{56}$ Interventions that encourage uptake adherence with COVID-19 vaccine programs will become increasingly important, especially in patients with NCDs over the coming months. Approval of the first effective vaccine by regulatory authorities in December 2020 followed on from data reported in Phase 1-3 clinical trials. ${ }^{57-60}$ Public health education programs on the safety of these vaccines and to counter public misperceptions or misunderstandings, together with planning to overcome the logistic challenges of vaccinating billions of people, are also vital. In the context of this 
paper, those who are likely to receive the vaccine first due to their high risk of severe COVID-19 are also those most at risk of having NCDs (ie, people aged 80 years or older).

The complexity of adherence and factors influencing it mean that to be effective, interventions designed to improve adherence need to be both multifaceted and tailored to the individual. ${ }^{61,62}$ Furthermore, while adherence has been improved by a range of interventions in the shortterm, relatively few studies have demonstrated long-term improvements in adherence. ${ }^{62,63} \mathrm{~A}$ one-size-fits-all approach will not work. For example, the patient's medical condition and history, health system factors that can impact access to medicines, social and economic situation, and educational level, all need to be taken into account. ${ }^{62}$ For example in Spain, health coverage is universal and all patients have access to medicines. The strategies in Spain are, therefore, more focused on personal, social, and educational issues rather than resolving problems with access to medications for example, which are more important in countries where access is limited. The importance of patient-centric healthcare has been recognized as key to many aspects of healthcare. For example, the Institute of Medicine has recognized that all healthcare should be safe, effective, timely, efficient, and equitable. ${ }^{64}$ Healthcare should also be centered around the patient. ${ }^{64}$ Good adherence with medications used to manage NCDs fulfills many of these requirements in that it is effective, safe, timely, and efficient. Furthermore, a multidisciplinary team needs to include those with clinical expertise, experience in patient engagement, behavioral change, program design and implementation, and service delivery.

Patients with NCDs often need to dedicate significant time, energy, and resources to dealing with multiple medical appointments and treatments. Therefore, it is important not to discourage the patient who is not adhering with their treatment regimen. The prescriber should instead use a patient-centric approach with non-adherence used as an opportunity to support the patient and find solutions by empowering and motivating them. ${ }^{34}$ Systems using artificial intelligence (AI) can aid patients with the scheduling of medical appointments. ${ }^{65}$ Shared decision-making between the patient and prescriber, in which the patient's values and preferences are effectively integrated, can improve adherence with medications used to treat NCDs. ${ }^{66}$

Both during and post-COVID-19 there is an urgent need for a strategic approach to improve adherence, using a combination of a patient-centric approach and any digital and mobile technology available today.
Continuous adherence with medications used to manage NCDs requires a significant and consistent behavioral change; therefore, the most effective methods to improve adherence are those that engage, coach, and educate the patient.

\section{The Use of RWD and RWE and Applying Digital Technology to Study and Improve Adherence}

A variety of different data sources and study designs can be used to measure adherence with medications. The application of $\mathrm{AI}$ and robotic process automation can help with the collection, collation, integration, and analysis of data from disparate sources. ${ }^{67}$ Real-world data (RWD) and real-world evidence (RWE) are becoming increasingly important in healthcare, across a broad range of different areas including regulatory submissions for medication label expansions and discussions around pricing and the budgetary impact of medications. ${ }^{67-77}$ RWD and RWE have been utilized for many decades to evaluate the effects of poor adherence (or conversely the benefits of improving adherence) with medications used to manage NCDs. ${ }^{33,36-38,78}$

During 2020, RWD and RWE have been at the forefront of the efforts to address the healthcare challenges of the COVID-19 pandemic. ${ }^{79}$ For example, there are now a number of national and international data "dashboards" providing real-time information on the incidence, prevalence, and mortality due to COVID-19. ${ }^{7,80,81}$ Information used in tracking and tracing those infected with COVID19 and on the availability of hospital beds for patients with severe COVID-19 are also vital to managing the pandemic.

Telemedicine has been used for over 30 years to help improve adherence with medications used to manage NCDs. For example, a study published in 1991 demonstrated the benefits of a health educational program that included a telephone call during which the patient was asked about their experiences with the antihypertensive medication, and reminded about the importance of adherence. ${ }^{82}$ More recently, telemedicine as part of a collaborative care program has been demonstrated to improve adherence with antidepressants in patients with depression. ${ }^{83}$ However, not every type of intervention implemented over the years has produced demonstrable outcomes and benefits. ${ }^{84}$ The increased availability and use of tablets, laptops, and in particular smartphones means that telemedicine is more accessible than in the 
past. The terms telehealth and mHealth are often used to describe the use of these devices for healthcare purposes. ${ }^{85}$ These devices also permit video-calls, which can replace some patient and clinician face-to-face consultations. This has been particularly important during the COVID-19 pandemic when "stay at home" orders have been common. ${ }^{86}$ Also, clinically vulnerable patients may be reluctant to visit hospitals due to their concerns about contracting COVID-19. Experience using these new technologies in practice will help to develop a catalog of the more useful approaches, which can be used either alone or in combination.

During the COVID-19 pandemic, access to telemedicine has been increased for patients, along with other improvements to patient access, to help ensure continuity of care across a variety of different US patient groups. For example, access to clinicians via telehealth has been provided to those with NCDs such as diabetes. ${ }^{87}$ Interactions between patients and their HCPs during the COVID-19 pandemic are now commonly conducted online rather than in person. ${ }^{88}$ Free mental health counseling via telemedicine has been provided by at least one US health insurance company. ${ }^{89}$

Other important modifications in healthcare due to the pandemic have included the loosening of access restrictions for medications used to manage NCDs to ensure continuity of care. These changes have included lowering the out of pocket co-pay costs for medications used to treat NCDs such as insulin, increasing the delivery options for these medications, eg, mail or other forms of home deliveries, and widening access to glucose monitors. ${ }^{87,89}$ Large US health insurers have also waived co-pays for tests used to diagnose COVID19 and for hospitalizations with COVID-19, as well as the treatment choices. ${ }^{90}$ Furthermore, schemes aimed at ensuring that those who have lost their healthcare coverage due to the pandemic have access to discounts on prescriptions have also been put in place. ${ }^{91}$ These modifications are aimed at indirectly improving the treatment of COVID-19 as well as overall health.

A review of studies designed to improve adherence with antihypertensive and lipid-lowering medications demonstrated that, in general, more involved and timeconsuming interventions such as case management are more effective than simpler interventions such as reminders to take medications or refill prescriptions. $^{92}$ Interventions that include a team-based approach to help co-ordinate care are often most successful. ${ }^{62,93}$ For example, a study using this collaborative approach achieved a $15 \%$ increase in adherence in patients discharged from hospital following a hospitalization for acute coronary syndrome. ${ }^{94}$ However, the more involved interventions and programs were not surprisingly more costly than the simpler ones. ${ }^{92}$ The wider availability of RWD and technology such as connected devices ${ }^{67}$ means that there are now many more means of interacting with patients with NCDs, and potentially improving their adherence. Some of the alterations to medical practice noted above, such as telemedicine and ehealth, seem likely to stay in place long after the pandemic. These technologies can therefore be utilized to provide a patient-centric approach to improving adherence and persistence. For example, e-prescribing software can be used to monitor prescription patterns such as scripts that are unfilled versus those that are filled and dispensed. ${ }^{62}$ This information could be shared and discussed with the patient during a consultation and in so doing, help to identify the patients' individual barriers to adherence and then collaboratively come up with solutions to overcome these barriers. ${ }^{62}$ Home measurements of BP can be used in a similar manner, if the patient is able to upload their BP measurements into their ehealth records. ${ }^{62}$

The potential for using RWD is now immense, with one large provider of RWD having access to information from over 1.1 billion patients. ${ }^{95,96}$ Not all of these 1.1 billion patients will have NCDs or the risk factors for NCDs. However, given the observation that NCDs are collectively responsible for nearly $70 \%$ of deaths worldwide, ${ }^{96}$ a substantial proportion of these patients have or will develop NCDs. This, therefore, suggest RWD will continue to play a vital role in improving the management of NCDs. ${ }^{74}$ Access to this information means that a customizable adherence dashboard can be created. This system allows queries regarding specific regions and individual medications to be addressed (Figure 5). In addition, there are many key areas for future research that may help improve adherence and persistence with medications used to manage NCDs. These include additional studies utilizing RWD and RWE to identify predictors of adherence (or conversely predictors of poor adherence), and for the assessment of interventions designed to improve adherence and persistence. The information gleaned from these studies could then be used to target interventions and educational initiatives to both healthcare stakeholders and patients on the importance of adherence in managing NCDs in the COVID era and beyond. There have been many changes to medical practice in the days since the 


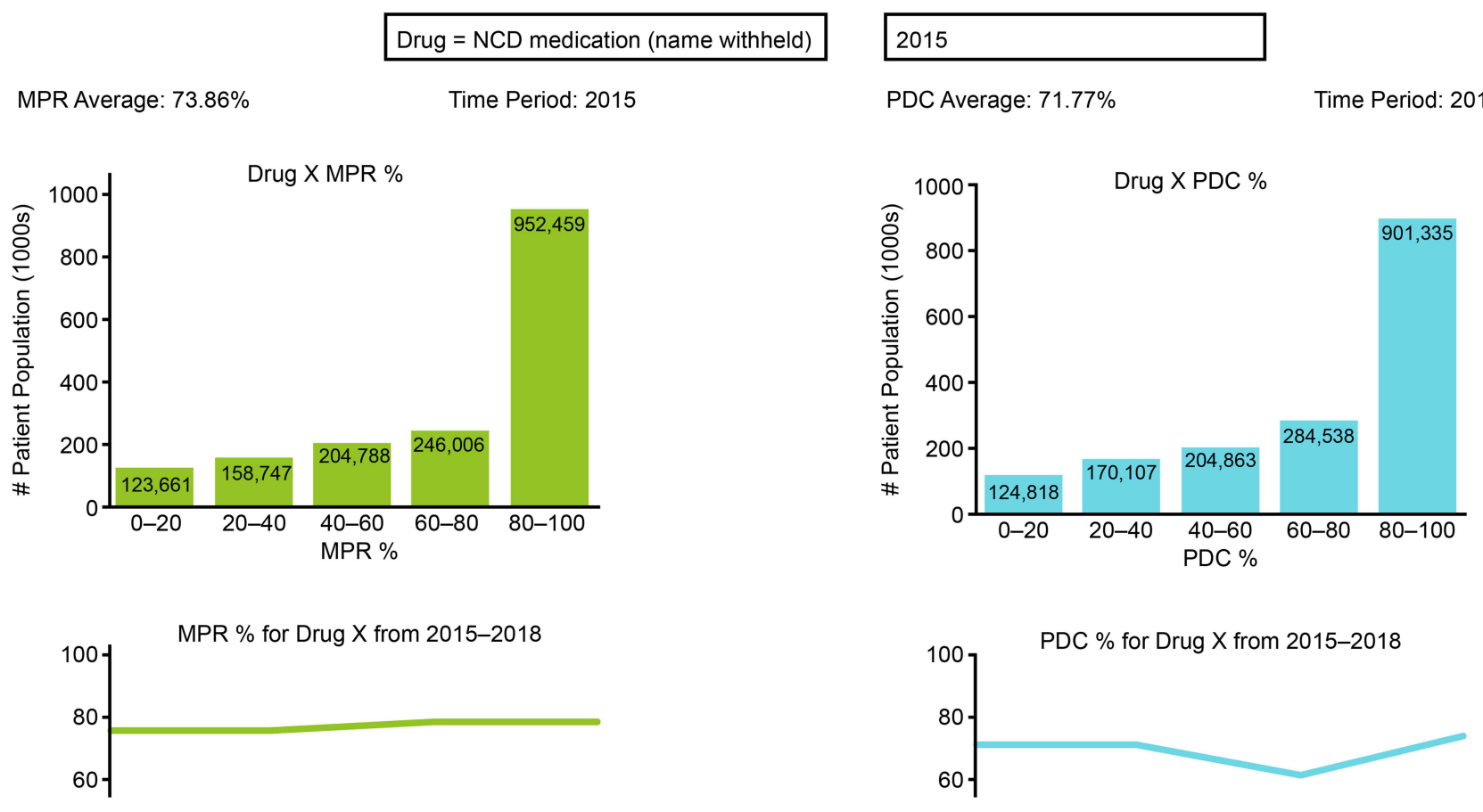

Figure 5 The use of RWD to monitor adherence and persistence over time - an example adherence dashboard.

Abbreviations: MPR, medical possession ratio; PDC, percentage of days covered; RWD, real-world data.

COVID-19 pandemic began, such as the increased use of telemedicine, AI, and other digital therapeutics. The increased uptake and investment of time and know-how into these technologies may also help drive innovative ways to monitor and provide targeted approaches to improve adherence and persistence via similar channels, in the post-COVID era.

\section{Adherence: A Spanish Example}

The potential impact of improving adherence with medications used in the management of NCDs in Spain is now being studied in detail. The burden of NCDs in Spain is considerable, with NCDs being responsible for $91 \%$ of all deaths (Figure 6; with 28\% due to CVD and 26\% due to cancer). ${ }^{3}$ Furthermore, adherence to medications used in the management of CV risk in Spain is low $(\sim 50 \%)$. Therefore, there is substantial room for improvement in adherence to $\mathrm{CV}$ medications, which could have a sizable benefit to those at risk of a CVD event, and in the Spanish healthcare system as a whole. Indeed, a Markov model validated by the Spanish Society of Community Pharmacy and the Spanish Society of Cardiology predicts that a $1 \%$ increase in adherence with therapies used to manage $\mathrm{CV}$ risk could prevent $1,100 \mathrm{CV}$ events, 1,200 deaths, and reduce avoidable healthcare expenditure by $€ 11$ million. A $10 \%$ increase in adherence with therapies used to manage $\mathrm{CV}$ risk is predicted to prevent 7,650 CV events and 8,788 deaths together with avoidable healthcare costs of $€ 75$ million.

The healthcare system in Spain provides universal coverage. Therefore, interventions to improve adherence should focus on condition-, therapy-, and patient-related factors. A program specifically designed to improve adherence with medications used to manage $\mathrm{CV}$ risk has been designed and is in the process of being implemented (Figure 5). Earlier studies have shown that interventions involving multidisciplinary teams (physicians, pharmacists, and nurses) are often more successful than programs working with just one element of the healthcare system and can improve adherence by up to $\sim 15 \%$ after 6 months. ${ }^{62,93,94}$ This collaborative multidisciplinary approach is being utilized with the creation of Adherence Working Teams in the two autonomous communities, in which this study is being initiated; namely Andalusia and the Region of Murcia. These two regions are very different in size, with Andalusia being much larger than Murcia. The program will, therefore, provide data that will be applicable to many other regions in Spain.

The first phase of the program involves creating the Adherence Medical Working Teams, which focus on 


\section{A. Background}
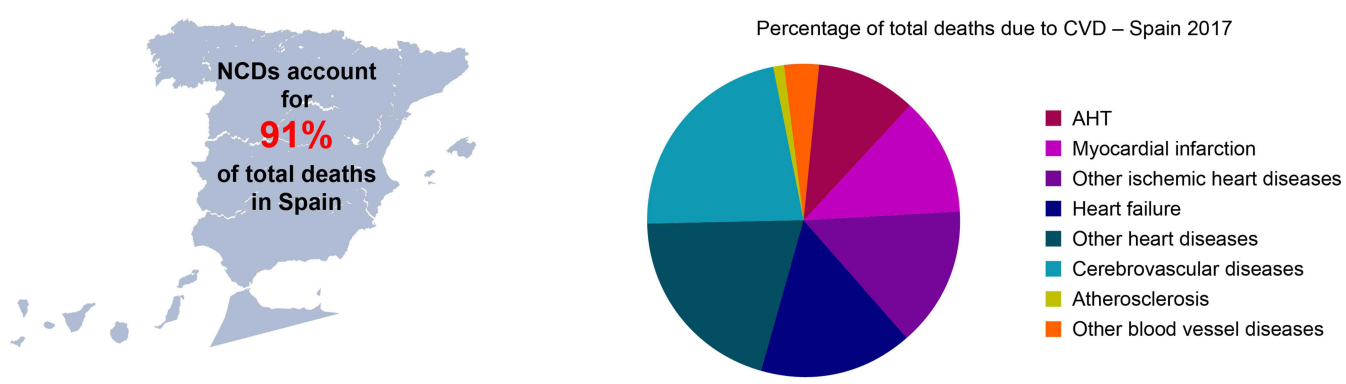

CVD is responsible for more deaths in Spain than any other cause

Main Goal: To increase therapeutic adherence in CVDs (currently $\mathbf{5 0 \%}$ )

\section{B. Main causes of nonadherence in Spain}

Spain HCS: Universal Coverage

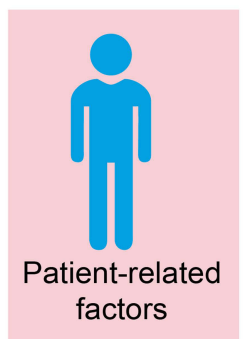

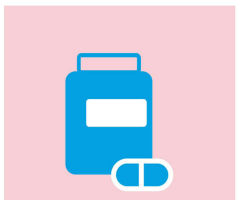

Therapy-related factors

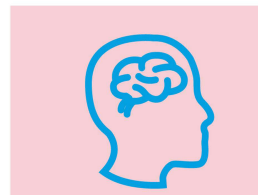

Condition-related factors

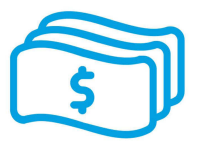

Socioeconomic

factors

Universal access to medicines

\section{ACs-OAT-Viatris Partnership}

Plan adjusted according to the characteristics of each autonomous community

\section{STEP 1}

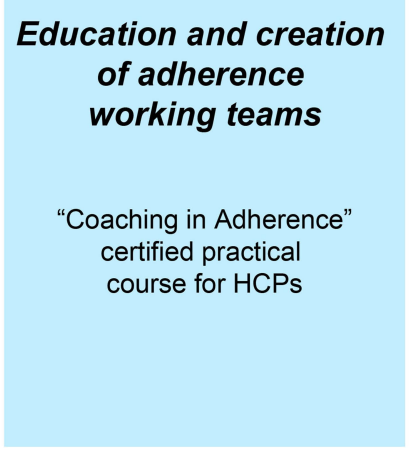

STEP 2

Implementation
initial phase
- Creation of the committee
of experts
- Establishment of baseline
metrics
- Segmentation by
non-adherence risk profile
- Selection of centers and
integration of health
professionals

\section{STEP 3}

\section{Implementation and data generation}

- Patient recruitment (Activare Platform)

- Follow-up reports (Activare Platform)

- Data generation

- Analysis and publication

Figure 6 Adherence in Spain, a case study.

Abbreviations: ACS-OAT, autonomous communities, observatory of adherence to therapy; AHT, arterial hypertension; CVD, cardiovascular disease; HCP, healthcare provider; HCS, healthcare system; NCD, noncommunicable disease. 
educating HCPs on the importance of adherence with therapies used to manage NCDs. The next phase involves identifying patients who are non-adherent to therapies and encouraging them to adhere with their CV-risk-reducing medications. Patient outcomes are then monitored, eg, adherence with therapy (percentage increase from baseline), satisfaction, and changes in QoL. The Markov model will be used to estimate the results of improved adherence on CV events, deaths, and avoidable healthcare expenditure. The initial results of this program are anticipated in late 2021.

Spain has recorded over 3.71 million cases of COVID-19 and more than 80,000 deaths from this condition as of June 9, $2021 .^{7}$ A focus on the management of NCDs and on improving adherence to medications and lifestyle improvements in particular is therefore more important than ever, given the link between NCDs and more severe COVID-19 symptoms.

\section{Conclusions}

The importance of adherence and persistence with medications that control NCDs has been a major focus for many years. However, the proportion of patients' remaining adherent and/or persistent with their medications long-term, remains very low, while the burden of NCDs is high and ever increasing. Interventions or programs designed to improve adherence are often time-consuming and costly. This is likely due to the many facets to adherence and persistence.

Recent evidence suggests a patient-centric approach to improve adherence is critically important to ensure patients are both motivated and empowered. Novel technologybased methods (eg, eHealth and mHealth) to promote and measure adherence and persistence are now available.

Relatively early during the COVID-19 pandemic, studies evaluating risk factors for poor prognosis among patients with COVID-19 demonstrated that those with NCDs were at a greater risk of poor clinical outcomes than those without NCDs. ${ }^{9,12,14-18}$ Therefore, the urgent nature of the pandemic has provided fresh impetus to encourage those with NCDs to be more adherent with their medications, and with therapeutic lifestyle changes, ie, improved nutrition and taking more exercise. Over the coming months, huge efforts and finances will be channeled into initiatives to promote adherence to the vaccination programs. Those at highest risk of severe COVID-19, who are likely to receive the vaccine first, are also those most at risk of having NCDs. PostCOVID-19, eHealth and $m$ Health will also likely remain relevant and see increased use and lasting impact on patients' access to care and their health. This may prove to be particularly important for those whose challenges to access lie in social determinants of health, which can impact not just the likelihood of having an
NCD or becoming infected with COVID-19 but also negatively impact a patient's access to healthcare, their adherence, and their persistence with treatment.

\section{Abbreviations}

AI, artificial intelligence; BP, blood pressure; COVID-19, COronaVIrus Disease 2019; CVD, cardiovascular disease; HCP, healthcare professionals; HCS, healthcare system; $\mathrm{HCT}$, healthcare team; MPR, medical possession ratio; NCD, noncommunicable disease; QoL, quality of life; PDC, percentage of days covered; RWD, real-world data; RWE, real-world evidence.

\section{Acknowledgments}

Medical writing support was provided by Jon Edwards, $\mathrm{PhD}$ of Engage Scientific (Horsham, UK) and was funded by Upjohn, a legacy Division of Pfizer and now merged with Mylan to be Viatris.

\section{Author Contributions}

All authors made substantial contributions to conception and design, acquisition of data, or analysis and interpretation of data; took part in drafting the article or revising it critically for important intellectual content; agreed to submit to the current journal; gave final approval of the version to be published; and agree to be accountable for all aspects of the work.

\section{Funding}

This work was funded by Upjohn a legacy Division of Pfizer, and now merged with Mylan to be Viatris.

\section{Disclosure}

All authors were full-time employees of Viatris (TAH, JES, DD, JPC, JSI and KHZ) when this paper was developed. TAH, JES, DD, JPC, JSI, and KHZ report receiving salaries from the Upjohn a Division of Pfizer, now merged with Mylan to be Viatris and are also stockholders of both Pfizer and Viatris. JSI is now a former employee of Viatris. The authors report no other conflicts of interest in this work.

\section{References}

1. World Health Organization. Noncommunicable diseases: key facts [website]. 2018. Available from: https://www.who.int/news-room/factsheets/detail/noncommunicable-diseases. Accessed November 27, 2019.

2. Ricardo CZ, Azeredo CM, Machado de Rezende LF, Levy RB. Cooccurrence and clustering of the four major non-communicable disease risk factors in Brazilian adolescents: analysis of a national school-based survey. PLoS One. 2019;14(7):e0219370. doi:10.1371/journal. pone. 0219370 
3. World Health Organization. Noncommunicable diseases (NCD) country profiles [website]. 2018. Available from: https:/www.who.int/publica tions/i/item/ncd-country-profiles-2018. Accessed August 7, 2020.

4. Bodenheimer T, Chen E, Bennett HD. Confronting the growing burden of chronic disease: can the U.S. health care workforce do the job? Health Aff (Millwood). 2009;28(1):64-74. doi:10.1377/hlthaff.28.1.64

5. World Health Organization. Global action plan for the prevention and control of noncommunicable diseases 2013-2020 [website]. 2020. Available from: https://apps.who.int/iris/handle/10665/274512. Accessed August 7, 2020.

6. ISPOR. Medication adherence and persistence, special interest group [website]. 2021. Available from: https://www.ispor.org/membergroups/special-interest-groups/medication-adherence-andpersistence. Accessed August 7, 2020.

7. World Health Organization. COVID-19 dashboard [website]. 2020. Available from: https://covid19.who.int/. Accessed October 15, 2020.

8. Azarpazhooh MR, Morovatdar N, Avan A, et al. COVID-19 pandemic and burden of non-communicable diseases: an ecological study on data of 185 countries. J Stroke Cerebrovasc Dis. 2020;29 (9):105089. doi:10.1016/j.jstrokecerebrovasdis.2020.105089

9. Docherty AB, Harrison EM, Green CA, et al. Features of 20133 UK patients in hospital with covid-19 using the ISARIC WHO Clinical Characterisation Protocol: prospective observational cohort study. BMJ. 2020;369:m1985. doi:10.1136/bmj.m1985

10. Hassan TA, Enrique Saenz J, Li JZ, Ducinskiene D, Imperato J, Zou KH. A confluence of acute and chronic diseases: risk factors among Covid-19 patients. Significance Magazine. 2020.

11. Onder G, Rezza G, Brusaferro S. Case-fatality rate and characteristics of patients dying in relation to COVID-19 in Italy. JAMA. 2020;323 (18):1775-1776. doi:10.1001/jama.2020.4683

12. Richardson S, Hirsch JS, Narasimhan M, et al. Presenting characteristics, comorbidities, and outcomes among 5700 patients hospitalized with COVID-19 in the New York City Area. JAMA. 2020;323 (20):2052-2059. doi:10.1001/jama.2020.6775

13. Yang C, Jin Z. An acute respiratory infection runs into the most common noncommunicable epidemic-COVID-19 and cardiovascular diseases. JAMA Cardiol. 2020;5(7):743-744. doi:10.1001/jamacardio. 2020.0934

14. Bergman M, Jagannathan R, Narayan KMV. Nexus of COVID-19 and diabetes pandemics: global public health lessons. Diabetes Res Clin Pract. 2020;164:108215. doi:10.1016/j.diabres.2020.108215

15. Hernandez-Galdamez DR, Gonzalez-Block MA, Romo-Duenas DK, et al. Increased risk of hospitalization and death in patients with COVID-19 and pre-existing noncommunicable diseases and modifiable risk factors in Mexico. Arch Med Res. 2020;51(7):683-689. doi:10.1016/j.arcmed.2020.07.003

16. Zali A, Gholamzadeh S, Mohammadi G, et al. Baseline characteristics and associated factors of mortality in COVID-19 patients; an analysis of 16000 cases in Tehran, Iran. Arch Acad Emerg Med. 2020;8(1):e70.

17. Williamson EJ, Walker AJ, Bhaskaran K, et al. Factors associated with COVID-19-related death using openSAFELY. Nature. 2020;584 (7821):430-436. doi:10.1038/s41586-020-2521-4

18. Iftimie S, Lopez-Azcona AF, Vicente-Miralles M, et al. Risk factors associated with mortality in hospitalized patients with SARS-CoV-2 infection. A prospective, longitudinal, unicenter study in Reus, Spain. PLoS One. 2020;15(9):e0234452. doi:10.1371/journal.pone.0234452

19. World Health Organization. The impact of the COVID-19 pandemic on noncommunicable disease resources and services: results of a rapid assessment [website]. 2020. Available from: https://www. who.int/publications/i/item/ncds-covid-rapid-assessment. Accessed December 18, 2020.

20. Hariyanto TI, Kurniawan A. Metformin use is associated with reduced mortality rate from coronavirus disease 2019 (COVID-19) infection. Obes Med. 2020;19:100290. doi:10.1016/j.obmed.2020. 100290
21. Zhang XJ, Qin JJ, Cheng X, et al. In-hospital use of statins is associated with a reduced risk of mortality among individuals with COVID-19. Cell Metab. 2020;32(2):176-187. doi:10.1016/j.cmet.20 20.06.015

22. Zhu L, She ZG, Cheng X, et al. Association of blood glucose control and outcomes in patients with COVID-19 and pre-existing type 2 diabetes. Cell Metab. 2020;31(6):1068-1077. doi:10.1016/j.cmet.20 20.04.021

23. Ran J, Song Y, Zhuang Z, et al. Blood pressure control and adverse outcomes of COVID-19 infection in patients with concomitant hypertension in Wuhan, China. Hypertens Res. 2020;43(11):1267-1276. doi:10.1038/s41440-020-00541-w

24. Kayhan Tetik B, Gedik Tekinemre I, Tas S. The effect of the COVID-19 pandemic on smoking cessation success. J Community Health. 2021;46(3):471-475..

25. Marmot M, Bell R. Social determinants and non-communicable diseases: time for integrated action. BMJ. 2019;364:1251. doi:10.1136/ bmj.1251

26. Murray CJ, Kulkarni S, Ezzati M. Eight Americas: new perspectives on U.S. health disparities. Am J Prev Med. 2005;29(5 Suppl 1):4-10. doi:10.1016/j.amepre.2005.07.031

27. Brunt H, Barnes J, Jones SJ, Longhurst JWS, Scally G, Hayes E. Air pollution, deprivation and health: understanding relationships to add value to local air quality management policy and practice in Wales, UK. J Public Health (Oxf). 2017;39(3):485-497. doi:10.1093/ pubmed/fdw084

28. Abrams EM, Szefler SJ. COVID-19 and the impact of social determinants of health. Lancet Respir Med. 2020;8(7):659-661. doi:10.1016/S2213-2600(20)30234-4

29. Patel AP, Paranjpe MD, Kathiresan NP, Rivas MA, Khera AV. Race, socioeconomic deprivation, and hospitalization for COVID-19 in English participants of a national biobank. Int $J$ Equity Health. 2020;19(1):114. doi:10.1186/s12939-020-01227-y

30. Yancy CW. COVID-19 and African Americans. JAMA. 2020;323 (19):1891-1892. doi:10.1001/jama.2020.6548

31. Cramer JA, Roy A, Burrell A, et al. Medication compliance and persistence: terminology and definitions. Value Health. 2008;11 (1):44-47. doi:10.1111/j.1524-4733.2007.00213.x

32. Jimmy B, Jose J. Patient medication adherence: measures in daily practice. Oman Med J. 2011;26(3):155-159. doi:10.5001/omj.2011.38

33. Elliott WJ, Plauschinat CA, Skrepnek GH, Gause D. Persistence, adherence, and risk of discontinuation associated with commonly prescribed antihypertensive drug monotherapies. J Am Board Fam Med. 2007;20(1):72-80. doi:10.3122/jabfm.2007.01.060094

34. Dube C. Concordance: a partnership in medicine-taking. Health Expect. 2010;13(1):108-110. doi:10.1111/j.1369-7625.2008.00526.x

35. World Health Organization. Adherence to long-term therapies [website]. 2003. Available from: https://www.who.int/chp/knowl edge/publications/adherence_full_report.pdf. Accessed July 21, 2021.

36. Chapman RH, Benner JS, Petrilla AA, et al. Predictors of adherence with antihypertensive and lipid-lowering therapy. Arch Intern Med. 2005;165(10):1147-1152. doi:10.1001/archinte.165.10.1147

37. Ho PM, Rumsfeld JS, Masoudi FA, et al. Effect of medication nonadherence on hospitalization and mortality among patients with diabetes mellitus. Arch Intern Med. 2006;166(17):1836-1841. doi:10.1001/archinte.166.17.1836

38. Ho PM, Magid DJ, Shetterly SM, et al. Medication nonadherence is associated with a broad range of adverse outcomes in patients with coronary artery disease. Am Heart J. 2008;155(4):772-779. doi:10. 1016/j.ahj.2007.12.011

39. Ofori-Asenso R, Sahle BW, Chin KL, et al. Poor adherence and persistence to sodium glucose co-transporter 2 inhibitors in real-world settings: evidence from a systematic review and meta-analysis. Diabetes Metab Res Rev. 2021;37:e3350. doi:10. 1002/dmrr.3350 
40. DiMatteo MR. Variations in patients' adherence to medical recommendations: a quantitative review of 50 years of research. Med Care. 2004;42(3):200-209. doi:10.1097/01.mlr.0000114908.90348.f9

41. Ofori-Asenso R, Jakhu A, Zomer E, et al. Adherence and persistence among statin users aged 65 years and over: a systematic review and meta-analysis. J Gerontol A Biol Sci Med Sci. 2018;73(6):813-819. doi:10.1093/gerona/glx169

42. Osterberg L, Blaschke T. Adherence to medication. $N$ Engl J Med. 2005;353(5):487-497. doi:10.1056/NEJMra050100

43. Khan R, Socha-Dietrich K. Investigating in medication adherence improves health outcomes and health system efficiency: adherence to medicines for diabetes, hypertension, and hyperlipidemia. OECD Health Working Papers No 105. 2018.

44. Brown MT, Bussell JK. Medication adherence: WHO cares? Mayo Clin Proc. 2011;86(4):304-314. doi:10.4065/mcp.2010.0575

45. Cutler RL, Fernandez-Llimos F, Frommer M, Benrimoj C, GarciaCardenas V. Economic impact of medication non-adherence by disease groups: a systematic review. BMJ Open. 2018;8(1):e016982. doi:10.1136/bmjopen-2017-016982

46. Iuga AO, McGuire MJ. Adherence and health care costs. Risk Manag Healthc Policy. 2014;7:35-44. doi:10.2147/RMHP.S19801

47. Viswanathan M, Golin CE, Jones CD, et al. Interventions to improve adherence to self-administered medications for chronic diseases in the United States: a systematic review. Ann Intern Med. 2012;157 (11):785-795. doi:10.7326/0003-4819-157-11-201212040-00538

48. Vrijens B, De Geest S, Hughes DA, et al. A new taxonomy for describing and defining adherence to medications. $\mathrm{Br} \mathrm{J}$ Clin Pharmacol. 2012;73(5):691-705. doi:10.1111/j.1365-2125.2012.04 167.x

49. Alfian SD, Sukandar H, Lestari K, Abdulah R. Medication adherence contributes to an improved quality of life in type 2 diabetes mellitus patients: a cross-sectional study. Diabetes Ther. 2016;7(4):755-764. doi:10.1007/s13300-016-0203-x

50. Pringle JL, Boyer A, Conklin MH, McCullough JW, Aldridge A. The Pennsylvania Project: pharmacist intervention improved medication adherence and reduced health care costs. Health Aff (Millwood). 2014;33(8):1444-1452. doi:10.1377/hlthaff.2013.1398

51. Kardas P, Lewek P, Matyjaszczyk M. Determinants of patient adherence: a review of systematic reviews. Front Pharmacol. 2013;4:91. doi:10.3389/fphar.2013.00091

52. Adella Halim D, Kurniawan A, Agung FH, et al. Understanding of young people about COVID-19 during early outbreak in Indonesia. Asia Pac J Public Health. 2020;32(6-7):363-365. doi:10.1177/ 1010539520940933

53. Lehane E, McCarthy G. Intentional and unintentional medication non-adherence: a comprehensive framework for clinical research and practice? A discussion paper. Int J Nurs Stud. 2007;44 (8):1468-1477. doi:10.1016/j.ijnurstu.2006.07.010

54. Petry NM, Rash CJ, Byrne S, Ashraf S, White WB. Financial reinforcers for improving medication adherence: findings from a meta-analysis. Am J Med. 2012;125(9):888-896. doi:10.1016/j. amjmed.2012.01.003

55. Freisling H, Viallon V, Lennon $\mathrm{H}$, et al. Lifestyle factors and risk of multimorbidity of cancer and cardiometabolic diseases: a multinational cohort study. BMC Med. 2020;18(1):5. doi:10.1186/ s12916-019-1474-7

56. Keenan J. Improving adherence to medication for secondary cardiovascular disease prevention. Eur J Prev Cardiol. 2017;24 (3_suppl):29-35. doi:10.1177/2047487317708145

57. Voysey M, Clemens SAC, Madhi SA, et al. Safety and efficacy of the ChAdOx1 nCoV-19 vaccine (AZD1222) against SARS-CoV-2: an interim analysis of four randomised controlled trials in Brazil, South Africa, and the UK. Lancet. 2021;397(10269):99-111.

58. Polack FP, Thomas SJ, Kitchin N, et al. Safety and efficacy of the BNT162b2 mRNA Covid-19 vaccine. $N$ Engl J Med. 2020;383 (27):2603-2615. doi:10.1056/NEJMoa2034577
59. US Food \& Drug Administration. Pfizer-BioNTech COVID-19 vaccine - emergency use authorization [website]. 2020. Available from: https:// www.fda.gov/emergency-preparedness-and-response/coronavirusdisease-2019-covid-19/pfizer-biontech-covid-19-vaccine. Accessed December 13, 2020.

60. Medicines \& Healthcare Products Regulatory Agency. Decision: conditions of authorisation for Pfizer/BioNTech COVID-19 vaccine [website]. 2020. Available from: https://www.gov.uk/government/pub lications/regulatory-approval-of-pfizer-biontech-vaccine-for-covid -19/conditions-of-authorisation-for-pfizerbiontech-covid-19-vaccine. Accessed December 13, 2020.

61. Bosworth HB, Granger BB, Mendys P, et al. Medication adherence: a call for action. Am Heart J. 2011;162(3):412-424. doi:10.1016/j. ahj.2011.06.007

62. Neiman AB, Ruppar T, Ho M, et al. CDC Grand Rounds: improving medication adherence for chronic disease management - innovations and opportunities. MMWR Morb Mortal Wkly Rep. 2017;66 (45):1248-1251. doi:10.15585/mmwr.mm6645a2

63. Demonceau J, Ruppar T, Kristanto P, et al. Identification and assessment of adherence-enhancing interventions in studies assessing medication adherence through electronically compiled drug dosing histories: a systematic literature review and meta-analysis. Drugs. 2013;73(6):545-562. doi:10.1007/s40265-013-0041-3

64. Agency for Healthcare Research and Quality (AHRQ). Organizing quality measures by domains of health care quality [website]. 2016. Available from: https://www.ahrq.gov/talkingquality/translate/orga nize/quality-domain.html. Accessed November 3, 2020.

65. Matulis JC, McCoy R. Patient-centered appointment scheduling: a call for autonomy, continuity, and creativity. $J$ Gen Intern Med. 2021;36(2):511-514. doi:10.1007/s11606-020-06058-9

66. Yamane SS, De Gagne JC, Riggs A, Kimberly GD, Holye M. Assessment of a patient-centered initiative to improve hypertension management for adults with comorbid type 2 diabetes at a free clinic in the rural south. Nurs Forum. 2020;55(3):348-355. doi:10.1111/nuf.12434

67. Zou KH, Imperato J, Potkar CN, Sethi N, Edwards J, Ray A. Harnessing real-world data for regulatory use and applying innovative applications. J Multidiscip Healthc. 2020;13:671-679. doi:10.2147/JMDH.S262776

68. US Food and Drug Administration. Use of real-world evidence to support regulatory decision-making for medical devices: guidance for industry and food and drug administration staff. Docket no. FDA-2016-D-2153 [website]. 2017. Available from: https://www. fda.gov/regulatory-information/search-fda-guidance-documents/usereal-world-evidence-support-regulatory-decision-making-medicaldevices. Accessed February 5, 2020.

69. US Food and Drug Administration. Framework for FDA's real-world evidence program [website]. 2018. Available from: https:/www.fda. gov/media/120060/download. Accessed November 21, 2019.

70. US Food and Drug Administration. Real-world evidence [website]. 2019. Available from: https://www.fda.gov/science-research/science-andresearch-special-topics/real-world-evidence. Accessed December 19, 2019.

71. US Food and Drug Administration. Submitting documents using real-world data and real-world evidence to FDA for drugs and biologics: guidance for industry. Docket no. 2019-09529 [website]. Center for Drug Evaluation and Rresearch, Center for Biologics Evaluation and Research; 2019. Available from: https://www.fda. gov/regulatory-information/search-fda-guidance-documents/submit ting-documents-using-real-world-data-and-real-world-evidence-fdadrugs-and-biologics-guidance. Accessed February 5, 2020.

72. European Medicines Agency. Regulatory perspective on real world evidence (RWE) in scientific advice. EMA human scientific committees' working parties with patients' and consumers' organisations (PCWP) and healthcare professionals' organisations (HCPWP) [website]. 2018. Available from: https://www.ema.europa.eu/en/documents/presentation/ presentation-regulatory-perspective-real-world-evidence-rwe-scientific -advice-emas-pcwp-hcpwp-joint_en.pdf. Accessed December 19, 2019. 
73. Fralick M, Kesselheim AS, Avorn J, Schneeweiss S. Use of health care databases to support supplemental indications of approved medications. JAMA Intern Med. 2018;178(1):55-63. doi:10.1001/ jamainternmed.2017.3919

74. Zou KH, Li JZ, Salem LA, Imperato J, Edwards J, Ray A. Harnessing real-world evidence to reduce the burden of noncommunicable disease: health information technology and innovation to generate insights. Health Serv Outcomes Res Methodol. 2020;1-13. doi:10.1007/s10742-020-00223-7

75. Baker CL, Ding Y, Ferrufino CP, Kowal S, Tan J, Subedi P. A cost-benefit analysis of smoking cessation prescription coverage from a US payer perspective. Clinicoecon Outcomes Res. 2018;10:359-370. doi:10.2147/CEOR.S165576

76. Garry EM, Schneeweiss S, Eapen S, et al. Actionable real-world evidence to improve health outcomes and reduce medical spending among risk-stratified patients with diabetes. J Manag Care Spec Pharm. 2019;25(12):1442-1452. doi:10.18553/jmcp.2019.25.12.1442

77. US Food \& Drug Administration. Real-world data (RWD) and real-world evidence (RWE) are playing an increasing role in health care decisions [website]. 2020. Available from: https:/www.fda.gov/ science-research/science-and-research-special-topics/real-worldevidence. Accessed December 14, 2020.

78. Chen ST, Huang ST, Shau WY, et al. Long-term statin adherence in patients after hospital discharge for new onset of atherosclerotic cardiovascular disease: a population-based study of real world prescriptions in Taiwan. BMC Cardiovasc Disord. 2019;19(1):62. doi:10.1186/s12872-019-1032-4

79. Abernethy AP. Advancing the science of real-world data to address the COVID-19 pandemic [website]. US Food \& Drug Administration; 2020. Available from: https://www.fda.gov/science-research/fda-grandrounds/advancing-science-real-world-data-address-covid-19-pandemic -09102020-09102020. Accessed December 14, 2020.

80. Centers for Disease Control and Prevention. Coronavirus disease 2019 (COVID-19) CDC COVID data tracker. United States COVID-19 cases and deaths by state [website]. 2020. Available from: https://covid.cdc.gov/covid-data-tracker/\#cases_casesper100k last7days. Accessed December 15, 2020.

81. Johns Hopkins University of Medicine Coronavirus Resource Center. COVID-19 dashboard by the center for systems science and engineering (CSSE) at Johns Hopkins University (JHU) [website]. 2020. Available from: https://coronavirus.jhu.edu/map.html. Accessed December 15, 2020 .

82. Sclar DA, Chin A, Skaer TL, Okamoto MP, Nakahiro RK, Gill MA. Effect of health education in promoting prescription refill compliance among patients with hypertension. Clin Ther. 1991;13(4):489-495.

83. Deen TL, Fortney JC, Pyne JM. Relationship between satisfaction, patient-centered care, adherence and outcomes among patients in a collaborative care trial for depression. Adm Policy Ment Health. 2011;38(5):345-355. doi:10.1007/s10488-010-0322-z

84. Piette JD, Aikens JE, Trivedi R, et al. Depression self-management assistance using automated telephonic assessments and social support. Am J Manag Care. 2013;19(11):892-900.
85. World Health Organization. mHealth new horizons for health through mobile technologies [website]. 2011. Available from: https://www.who. int/goe/publications/goe_mhealth_web.pdf. Accessed January 27, 2021.

86. Wosik J, Fudim M, Cameron $\bar{B}$, et al. Telehealth transformation: COVID-19 and the rise of virtual care. J Am Med Inform Assoc. 2020;27(6):957-962. doi:10.1093/jamia/ocaa067

87. Centers for Medicare \& Medicaid Services. President Trump announces lower out of pocket insulin costs for Medicare's seniors [website]. CMS.gov; 2020. Available from: https://www.cms.gov/ newsroom/press-releases/president-trump-announces-lower-outpocket-insulin-costs-medicares-seniors. Accessed November 3, 2020.

88. Ghosh A, Gupta R, Misra A. Telemedicine for diabetes care in India during COVID19 pandemic and national lockdown period: guidelines for physicians. Diabetes Metab Syndr. 2020;14(4):273-276. doi:10.10 16/j.dsx.2020.04.001

89. AETNA. Extra benefits for you: enhanced coverage includes free counseling, COVID-19 testing and prescription home delivery [website]. 2020. Available from: https://www.aetna.com/individuals-families/memberrights-resources/covid19/benefits.html. Accessed November 5, 2020.

90. Gill LL. Paying for healthcare in the age of coronavirus [website]. 2020. Available from: https:/www.consumerreports.org/healthcare-costs/pay ing-for-healthcare-in-the-age-of-coronavirus/. Accessed November 5, 2020.

91. Queller D. Parachute Rx: peace of mind for uninsured Americans [website]. 2020. Available from: https://www.express-scripts.com/cor porate/articles/parachute-rx-peace-mind-uninsured-americans. Accessed November 5, 2020.

92. Chapman RH, Ferrufino CP, Kowal SL, Classi P, Roberts CS. The cost and effectiveness of adherence-improving interventions for antihypertensive and lipid-lowering drugs. Int J Clin Pract. 2010;64 (2):169-181. doi:10.1111/j.1742-1241.2009.02196.X

93. Zheng Y, Ding X, Guo Y, et al. Multidisciplinary management improves anxiety, depression, medication adherence, and quality of life among patients with epilepsy in eastern China: a prospective study. Epilepsy Behav. 2019;100(Pt A):106400. doi:10.1016/j.yebeh.2019.07.001

94. Ho PM, Lambert-Kerzner A, Carey EP, et al. Multifaceted intervention to improve medication adherence and secondary prevention measures after acute coronary syndrome hospital discharge: a randomized clinical trial. JAMA Intern Med. 2014;174 (2):186-193. doi:10.1001/jamainternmed.2013.12944

95. IQVIA. Harness the power of Real World Data [website]. 2021. Available from: https://www.iqvia.com/solutions/real-world-evi dence/real-world-data-and-insights . Accessed July 20, 2021.

96. World Health Organization. Noncommunicable diseases: Overview: [website] Available at: https:// www.who.int/health-topics/noncommu nicable-diseases\#tab=tab 1. Accessed, December 18, 2020

97. World Health Organization. Global Action Plan for the Prevention and Control of NCDs 2013-2020 [website]. 2021. Avaliable from: https:// www.who.int/publications/i/item/9789241506236. Accessed January 26,2021
Journal of Multidisciplinary Healthcare

\section{Publish your work in this journal}

The Journal of Multidisciplinary Healthcare is an international, peerreviewed open-access journal that aims to represent and publish research in healthcare areas delivered by practitioners of different disciplines. This includes studies and reviews conducted by multidisciplinary teams as well as research which evaluates the results or conduct of such teams or healthcare processes in general. The journal

Submit your manuscript here: https://www.dovepress.com/journal-of-inflammation-research-journal covers a very wide range of areas and welcomes submissions from practitioners at all levels, from all over the world. The manuscript management system is completely online and includes a very quick and fair peer-review system. Visit http://www.dovepress.com/testimonials. php to read real quotes from published authors. 